\title{
High-gradient near-quench-limit operation of superconducting Tesla-type cavities in scope of the International Linear Collider
}

\author{
Mathieu Omet, Hitoshi Hayano, Ayaka Kuramoto, Toshihiro Matsumoto, \\ Shinichiro Michizono, Takako Miura, and Feng Qiu \\ The Graduate University for Advanced Studies, Shonan Village, Hayama, Kanagawa 240-0193, Japan \\ and High Energy Accelerator Research Organization, 1-1 Oho, Tsukuba, Ibaraki 305-0801, Japan
}

(Received 20 February 2014; published 25 July 2014)

\begin{abstract}
We report the successful demonstration of an ILC-like high-gradient near-quench-limit operation at the Superconducting RF Test Facility at the High Energy Accelerator Research Organization (KEK) in Japan. Preparation procedures necessary for the accelerator operation were conducted, such as rf phase calibration, beam-based gradient calibration, and automated beam compensation. Test runs were performed successfully for nominal operation, high-loaded $\mathrm{Q}\left(\mathrm{Q}_{\mathrm{L}}\right)$ operation, and automated $\mathrm{P}_{\mathrm{k}} \mathrm{Q}_{\mathrm{L}}$ operation. The results are described in terms of the achieved precision and stabilities of gradients and phases.
\end{abstract}

DOI: 10.1103/PhysRevSTAB.17.072003

PACS numbers: $84.37 .+q, 84.40 . \mathrm{Fe}, 84.40 . \mathrm{Xb}$

\section{INTRODUCTION}

The International Linear Collider (ILC) [1] is a future high-luminosity linear electron-positron collider with an approximate length of $31 \mathrm{~km}$ [2]. Its purpose is highprecision investigation of the Higgs boson, dark matter, and probing of extra dimensions. ILC is based on $1.3 \mathrm{GHz}$ superconducting radio-frequency accelerating technology. The total number of superconducting cavities used will be approximately 16000 . The cavities will be driven in a configuration of one $10 \mathrm{MW}$ klystron per 39 cavities. They will be controlled by vector sum. The vector sum is the sum of the complex vectors (in I and Q or amplitude and phase, respectively) representing the accelerating fields in all cavities.

The Superconducting RF Test Facility (STF) is operated at the High Energy Accelerator Research Organization (KEK) in Japan in preparation of ILC. From April 2012 to March 2013 the quantum beam (QB) project [3] was conducted at STF. Its goal was the demonstration of high-brightness $\mathrm{x}$-ray creation by inverse laser Compton scattering. To this end a linear electron accelerator was set up. It consisted of two superconducting nine-cell TESLAtype L-band cavities driven by a single klystron in the distributed radio frequency scheme [4]. The cavities were operated by digital low level radio frequency (LLRF) control techniques $[5,6]$ in a $5 \mathrm{~Hz}$ pulsed mode. A simplified schematic of the LLRF control loop is shown in Fig. 1.

momet@post.kek.jp

Published by the American Physical Society under the terms of the Creative Commons Attribution 3.0 License. Further distribution of this work must maintain attribution to the author(s) and the published article's title, journal citation, and DOI.
The cavity signals were down-converted by mixing them with a local oscillator (LO) signal with a frequency of $1310 \mathrm{MHz}$. Next, they were filtered (LPF) and digitized by analog-to-digital converters (ADCs) on the $\mu \mathrm{TCA}$ board [5]. After a signal rotation (ADC ROT) and computation of the vector sum, the signal was filtered (cavsim IIR) and subtracted from the set table. Next, the proportional gain was applied and a base feed forward (FF) as well as beam FF tables were added. The rotated (DAC ROT) and digitalto-analog converted signal was filtered by an analog $400 \mathrm{kHz}$ low-pass filter in order to suppress the excitation of $8 \pi / 9$ modes [7]. The filtered signal was sent to the klystron, which drives both superconducting cavities. To this end the klystron output power was divided by a variable hybrid, which has a range from 0 to $-6 \mathrm{~dB}$ for the through port. The cavity phases were set individually by remotely controlled waveguide phase shifters. Since remote control of the coupler positions was not available, remotely controlled waveguide reflectors were used in order to adjust the loaded quality factors $\left(\mathrm{Q}_{\mathrm{L}}\right)$ of the cavities. The possible range in $\mathrm{Q}_{\mathrm{L}}$ was $2.5 \times 10^{6}$ to $5 \times 10^{7}$. Lorentz force detuning in the cavities was compensated dynamically by piezotuners, which were operated using a sine-wave voltage [8].

ILC will be operated in a pulsed mode with a repetition rate of $5 \mathrm{~Hz}$. Figure 2(a) shows typical cavity gradient shapes without beam, which can be divided into three phases; a filling phase, in which the cavity gradients are built up, a flattop phase, in which the cavity gradients are kept constant, and the decay phase, in which the cavity gradients decay. Commonly the beam is accelerated during the flattop phase. The nominal design gradient for the ILC main linacs is $31.5 \mathrm{MV} / \mathrm{m}$ for a beam current of $5.8 \mathrm{~mA}$ [2]. A cavity gradient spread of $20 \%$ around the average gradient is planned. A further requirement is the operation of all cavities with flat gradients at 5\% below their 


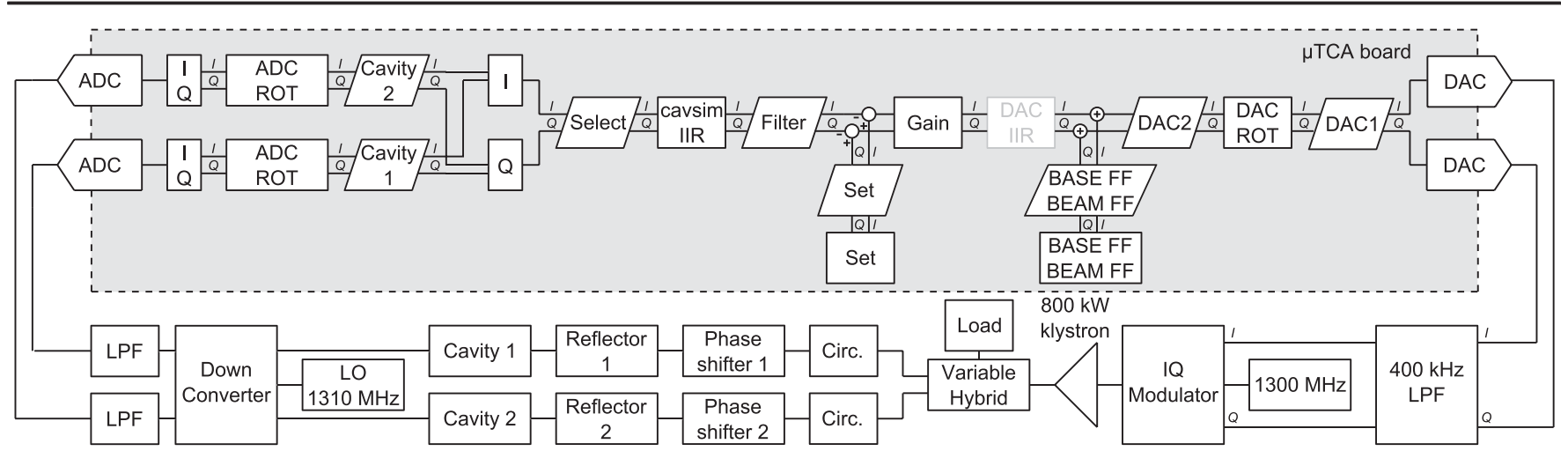

FIG. 1. Schematic of the digital LLRF feedback loop controlling two superconducting cavities at STF. Hardware and software components are represented by rectangles and the data channels accessible on the Micro Telecommunications Computing Architecture ( $\mu$ TCA) board via the Experimental Physics and Industrial Control System by rhombi.

respective quench limits, which must never be exceeded. This guarantees that the requirement for flat gradients is fulfilled at the same time. This requirement originates from the fact that cavity gradient tilts and rf fluctuations induce transverse beam orbit changes [9]. Nominal operation covers only the individual adjustment of the cavity driving powers $\mathrm{P}_{\mathrm{k}}$. In this operation mode, only nonflat flattops at different cavity gradients during beam transient can be achieved, as illustrated in Fig. 2. Solely by adjusting the driving power $\mathrm{P}_{\mathrm{k}}$ and the $\mathrm{Q}_{\mathrm{L}}$ value of each cavity individually $\left(\mathrm{P}_{\mathrm{k}} \mathrm{Q}_{\mathrm{L}}\right.$ operation) [10] both requirements, namely different gradients and flat flattops for all cavities, can be fulfilled. In order to establish such an operation, an automated beam compensation and a fully automated setting procedure for $\mathrm{P}_{\mathrm{k}} \mathrm{Q}_{\mathrm{L}}$ operation had to be developed and tested. The study presented builds up on a collaborative effort driven by the R\&D for ILC, to which researchers from several institutes across the world have contributed. A first theoretical approach to $\mathrm{P}_{\mathrm{k}} \mathrm{Q}_{\mathrm{L}}$ operation was published in 2007 [11]. A partial demonstration of such a kind of operation was performed during the $9 \mathrm{~mA}$ ILC tests at the free electron laser in Hamburg (FLASH) at Deutsches Elektronen-Synchrotron (DESY) in 2011 [12]. The present
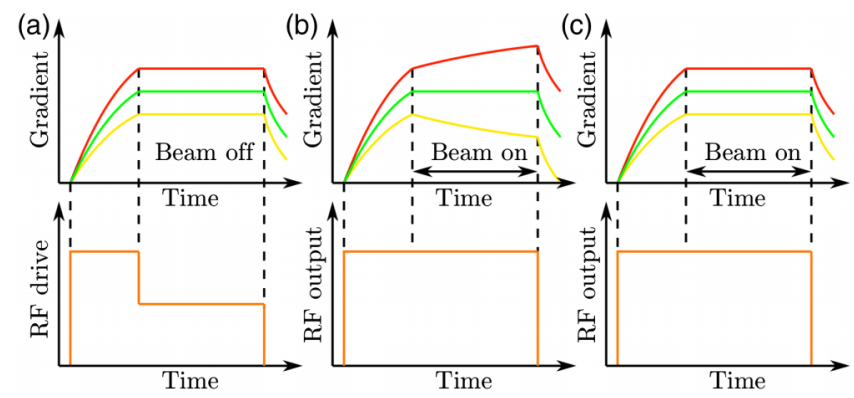

FIG. 2. Schematic of cavity 1 (yellow), cavity 2 (red), and vector-sum (green) gradients with corresponding rf output for (a) nominal operation without beam, (b) nominal operation with beam, and (c) $\mathrm{P}_{\mathrm{k}} \mathrm{Q}_{\mathrm{L}}$ operation with beam. study describes the development and performance of a full demonstration of a $\mathrm{P}_{\mathrm{k}} \mathrm{Q}_{\mathrm{L}}$ operation for the first time.

\section{IDENTIFICATION OF RF PHASE FOR ON-CREST ACCELERATION}

In the initial situation before operation, the rf phases of the cavities were random and needed to be calibrated for on-crest beam acceleration. For this calibration we used the cavity gradient changes induced by beam-loading effects [13]. From the cavity differential equation,

$$
\frac{d V_{\mathrm{cav}}}{d t}=-\omega_{1 / 2} V_{\mathrm{cav}}+R_{L} \omega_{1 / 2}\left(I_{g}-I_{b}\right),
$$

where $V_{\text {cav }}$ is the cavity voltage, $\omega_{1 / 2}$ the cavity half bandwidth, $R_{L}$ the loaded shunt impedance, $I_{g}$ the generator current, and $I_{b}$ the beam current, the condition of a constant beam phase at $\phi_{\text {beam }}=180^{\circ}$, and substituting $R_{L}$ and $\omega_{1 / 2}$, the beam-induced change in the cavity gradient is

$$
\Delta V_{\text {ind }}=\pi \frac{r}{Q} f_{0} I_{b} \cos \left(\phi_{\mathrm{rf}}\right) \Delta t,
$$

where $f_{0}$ is the resonance frequency, $\phi_{\mathrm{rf}}$ the $\mathrm{rf}$ phase, and $\Delta t$ the beam transient time. This shows that the change in the cavity gradient has a minimum at the rf phase for oncrest beam acceleration, where the phase difference between the beam and the rf phase is $\phi_{\mathrm{rf}}-\phi_{\text {beam }}=180^{\circ}$. By performing an rf phase scan during beam operation, the rf phase for the maximal cavity gradient drop can be found, and thus the rf phase for on-crest beam acceleration. This method was applied in an automated way individually to both cavities at STF. The result is shown in Fig. 3 in terms of the beam-induced change in the cavity gradient, corrected by the beam charge (ADC value/pC) versus the rf phase shift $\left({ }^{\circ}\right)$ for both cavities. The minima were determined by a sine-function fit. A low power measurement after the calibration showed that the deviation of the relative cavity rf phases set was better than $3^{\circ}$. 

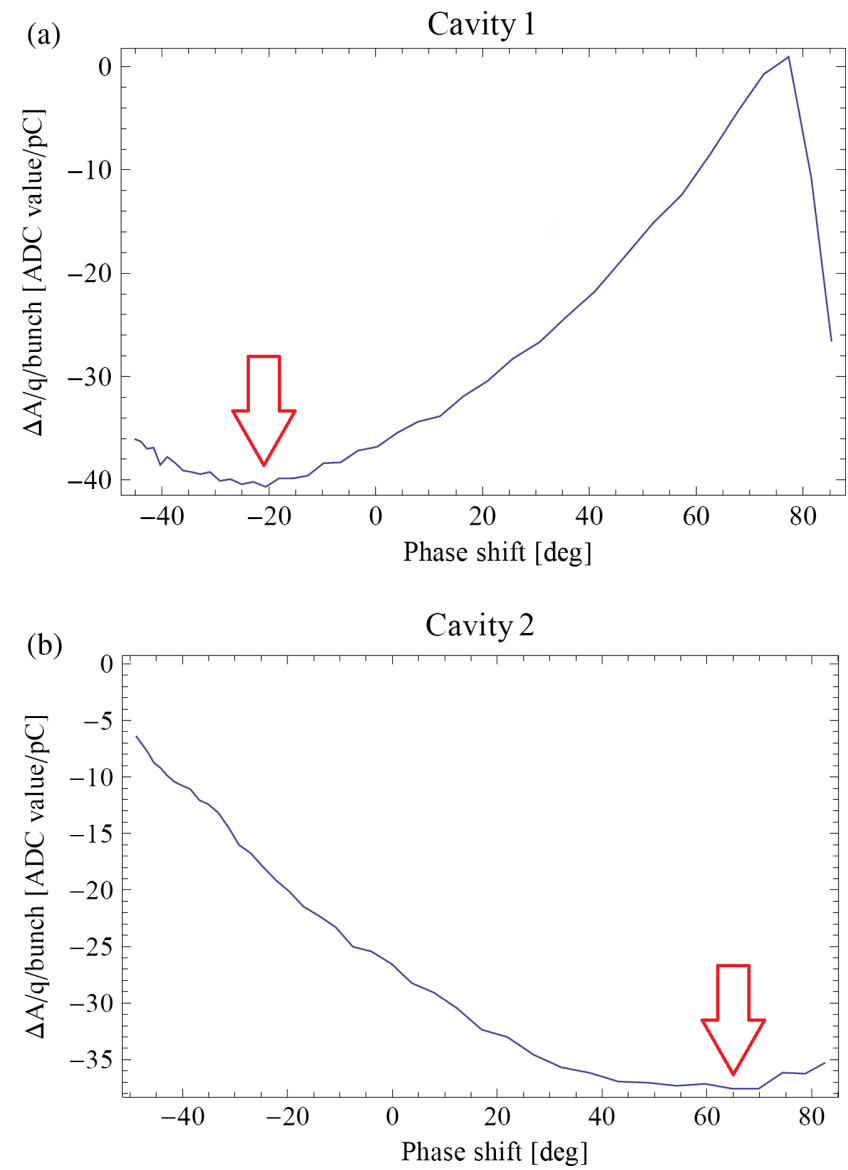

FIG. 3. Beam-induced change in the cavity gradient corrected by the beam charge (ADC value/pC) versus rf phase shift $\left(^{\circ}\right)$ for (a) cavity 1 and (b) cavity 2 . The red arrows indicate the locations of the minima.

\section{BEAM-BASED GRADIENT CALIBRATION}

To transform the picked-up, down-converted, and digitized cavity signals to a gradient, the ADC numbers had to be calibrated. For this calibration one can use beam-loading effects as described in the previous section. In the case of an on-crest beam acceleration, Eq. (2) can be simplified to

$$
\Delta V_{\text {ind }}=\pi \frac{r}{Q} f_{0} I_{b} \Delta t
$$

Figure 4 shows an example of a cavity gradient versus time with a beam transient of $31 \mu \mathrm{s}$. The average beam current was $9.9 \mathrm{~mA}$, which, according to Eq. (3), corresponds to a cavity gradient drop of $1.31 \mathrm{MV} / \mathrm{m}$. The actual drop was $1.26 \mathrm{MV} / \mathrm{m}$, which is within $3.8 \%$ of the estimated value. A linear correction function is determined by taking into account the estimated cavity gradient drop, the corresponding change in ADC counts, and the point of origin. This procedure was performed for the digitized pickup signals of both cavities.

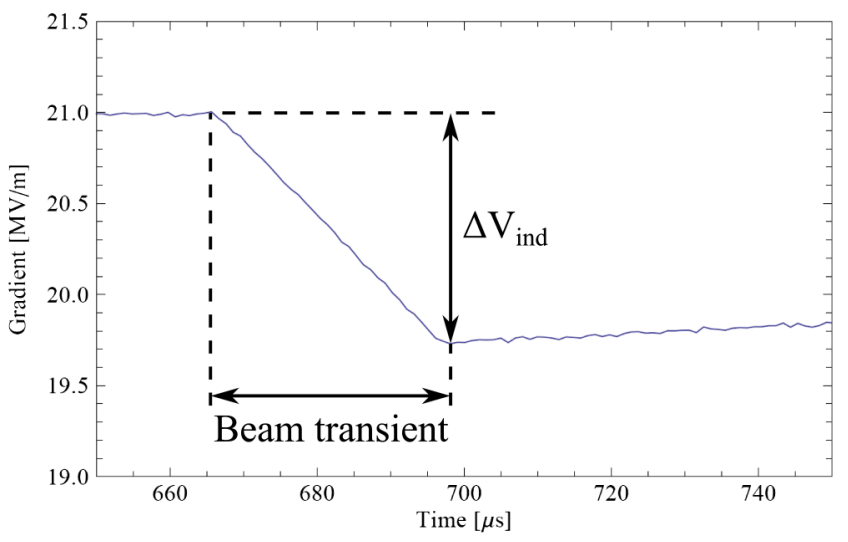

FIG. 4. Cavity 1 gradient $(\mathrm{MV} / \mathrm{m})$ versus time $(\mu \mathrm{s})$ during the flattop covering the beam transient, where the beam loading induced the gradient drop $\Delta \mathrm{V}_{\text {ind }}$.

\section{AUTOMATED BEAM COMPENSATION}

For the compensation of the beam-induced gradient drop, additional driving power had to be supplied during the beam transient. This was achieved by the addition of a beam FF table to the base FF table as shown in Fig. 5. Since the beam has a nonconstant structure over the whole pulse, a fully automated beam FF amplitude shape generation was established [10]. Figure 6 shows an example of an automatically generated beam FF table for a $5.5 \mathrm{~mA}$ beam with a pulse width of $308 \mu \mathrm{s}$. Due to beam current fluctuations during the generation procedure, the beam

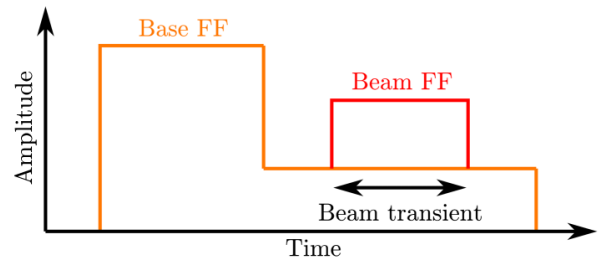

FIG. 5. Schematic of amplitude versus time for a base FF table (orange) and a beam FF table (red).

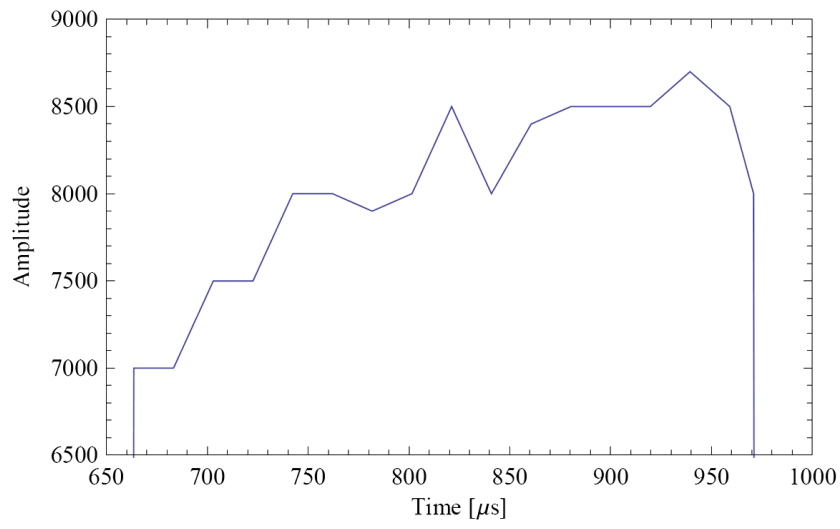

FIG. 6. Amplitude versus time $(\mu \mathrm{s})$ of an automatically generated beam FF table. 


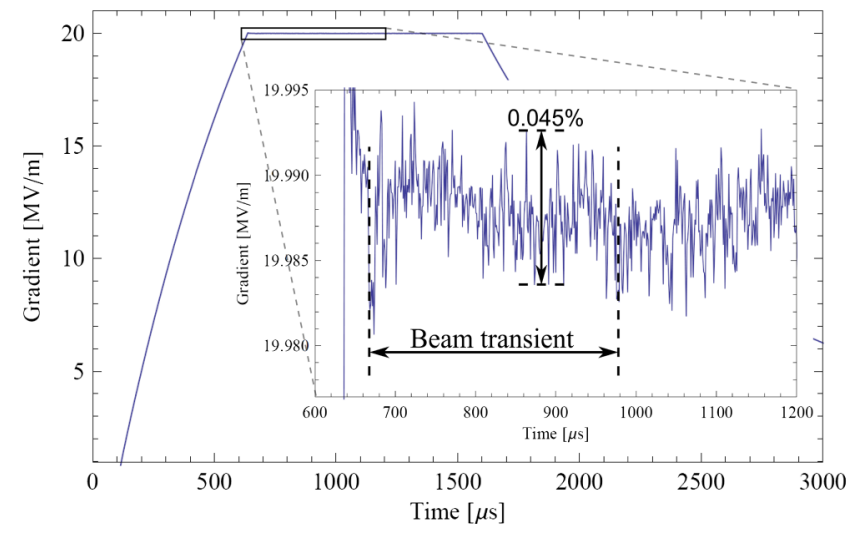

FIG. 7. Vector-sum gradient $(\mathrm{MV} / \mathrm{m})$ versus time $(\mu \mathrm{s})$ after automated generation of the beam FF table during nominal operation.

FF amplitude table is not smooth. Since the cavity itself acts like a low-pass filter, the high-frequency components of this structure are not an issue. Figure 7 shows the vectorsum gradient after the automated generation and the appliance of a beam FF table during nominal operation. The stabilities are presented in the next section (Table I).

\section{LONG-TIME NOMINAL OPERATION}

In the scope of the $\mathrm{QB}$ project, the nominal operation parameters were $\mathrm{V}_{\text {Cav1 }}=16 \mathrm{MV} / \mathrm{m}$ and $\mathrm{V}_{\mathrm{Cav} 2}=$ $24 \mathrm{MV} / \mathrm{m}$, with $\mathrm{Q}_{\mathrm{L}, \text { Cav1 }}=\mathrm{Q}_{\mathrm{L}, \text { Cav2 }}=3 \times 10^{6}$ and a filling time of $540 \mu \mathrm{s}[10,14]$. The beam compensation was automatically matched to the beam-pulse profile. The vector-sum stabilities during the beam transient achieved in a $1 \mathrm{~h}$ long-time run with and without a $6.6 \mathrm{~mA}$ beam with a pulse length of $615 \mu$ s are listed in Table I. A snapshot of the vector-sum gradient is shown in Fig. 7.

\section{HIGH QL OPERATION}

The operational cavity gradients at ILC will be $(31.5 \pm 20 \%) \mathrm{MV} / \mathrm{m}$. The variation of $20 \%$ in the gradient stems from the quench limits, which differ between cavities. This requires a $\mathrm{Q}_{\mathrm{L}}$ range from $3 \times 10^{6}$ to $10 \times 10^{6}$ [14]. Since $f_{1 / 2}=\frac{f_{0}}{2 Q_{\mathrm{L}}}$ the cavity bandwidth becomes narrow at high $\mathrm{Q}_{\mathrm{L}}$ values (e.g., $f_{1 / 2}=32.5 \mathrm{~Hz}$ at $Q_{\mathrm{L}}=2 \times 10^{7}$ and $f_{0}=1.3 \mathrm{GHz}$ ). Because of this,

TABLE I. Best snapshot vector-sum amplitude and phase stabilities under long-time nominal operation [root-mean-square (rms) values].

\begin{tabular}{lll}
\hline \hline Beam & $6.6 \mathrm{~mA}$ & Off \\
\hline$\Delta \mathrm{A} / \mathrm{A}$ vector sum & $0.009 \%$ & $0.008 \%$ \\
$\Delta \phi$ vector sum & $0.009^{\circ}$ & $0.008^{\circ}$ \\
\hline \hline
\end{tabular}

TABLE II. Best snapshot vector-sum amplitude and phase stabilities under high $\mathrm{Q}_{\mathrm{L}}$ operation (rms values).

\begin{tabular}{lll}
\hline \hline Beam & $6.1 \mathrm{~mA}$ & Off \\
\hline$\Delta \mathrm{A} /$ A cavity 1 & $0.12 \%$ & $0.03 \%$ \\
$\Delta \mathrm{A} /$ A cavity 2 & $0.16 \%$ & $0.03 \%$ \\
$\Delta \mathrm{A} /$ A vector sum & $0.011 \%$ & $0.008 \%$ \\
$\Delta \phi$ cavity 1 & $0.03^{\circ}$ & $0.03^{\circ}$ \\
$\Delta \phi$ cavity 2 & $0.03^{\circ}$ & $0.03^{\circ}$ \\
$\Delta \phi$ vector sum & $0.015^{\circ}$ & $0.014^{\circ}$ \\
\hline \hline
\end{tabular}

cavity detuning, induced by e.g., microphonics, is expected to become severe.

At KEK STF, the range of $\mathrm{Q}_{\mathrm{L}}$ values of both superconducting cavities is $2.5 \times 10^{6}$ to $5 \times 10^{7}$. For the demonstration of high $\mathrm{Q}_{\mathrm{L}}$ operation, the $\mathrm{Q}_{\mathrm{L}}$ values were set to $2 \times 10^{7}$. Because of this, the filling time was extended to $800 \mu \mathrm{s}$. Both cavity gradients were set to $20 \mathrm{MV} / \mathrm{m}$. In a $1 \mathrm{~h}$ long-time run with a $6.1 \mathrm{~mA}$ beam with a pulse length of $615 \mu \mathrm{s}$ and beam compensation and feedback (FB) turned on, amplitude and phase stabilities were achieved as listed in Table II. Figure 8 shows a snapshot of the vector-sum gradient. A run of 20 minutes at the same condition without beam was also performed. The corresponding stabilities are also listed in Table II.

The measured $\mathrm{Q}_{\mathrm{L}}$ values of both cavities during beam operation, which were determined by the evaluation of the cavity gradient decays, were on average $\mathrm{Q}_{\mathrm{L}, \mathrm{Cav} 1}=2.1 \times 10^{7}$ and $\mathrm{Q}_{\mathrm{L}, \mathrm{Cav} 2}=2.2 \times 10^{7}$. The aberrations from the set value originated from an imprecision of the setting method, since the $\mathrm{Q}_{\mathrm{L}}$ values in this region were very sensitive to even small changes in the waveguide reflector step motor positions. The Lorentz force detuning compensation was performed with a feed-forward excitation of the piezos. Figure 9(a) shows the detuning for both cavities during the $1 \mathrm{~h}$ time span. The corresponding histograms are shown in Fig. 9(b). The standard deviations are $\sigma_{\Delta f \text {,Cav1 }}=10.1 \mathrm{~Hz}$ and $\sigma_{\Delta f, \text { Cav2 }}=4.7 \mathrm{~Hz}$. This shows that the cryogenics

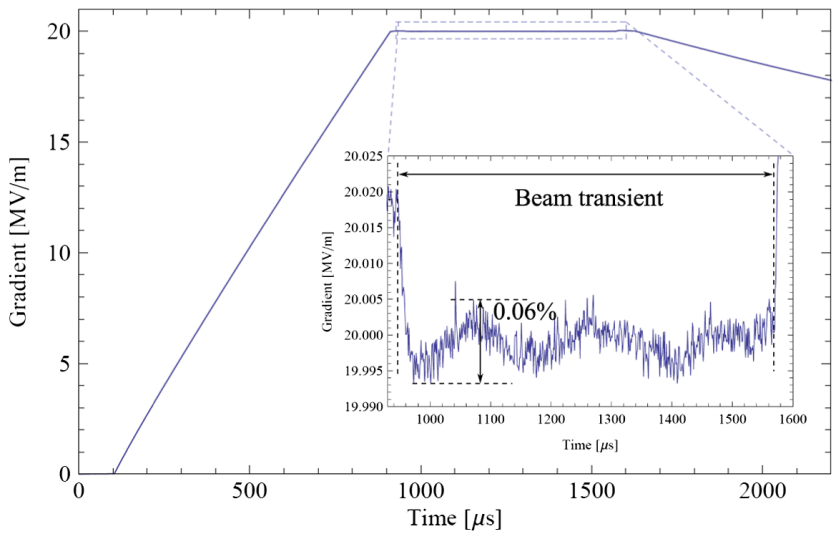

FIG. 8. Vector-sum gradient $(\mathrm{MV} / \mathrm{m})$ versus time (s) during high $\mathrm{Q}_{\mathrm{L}}$ operation. 

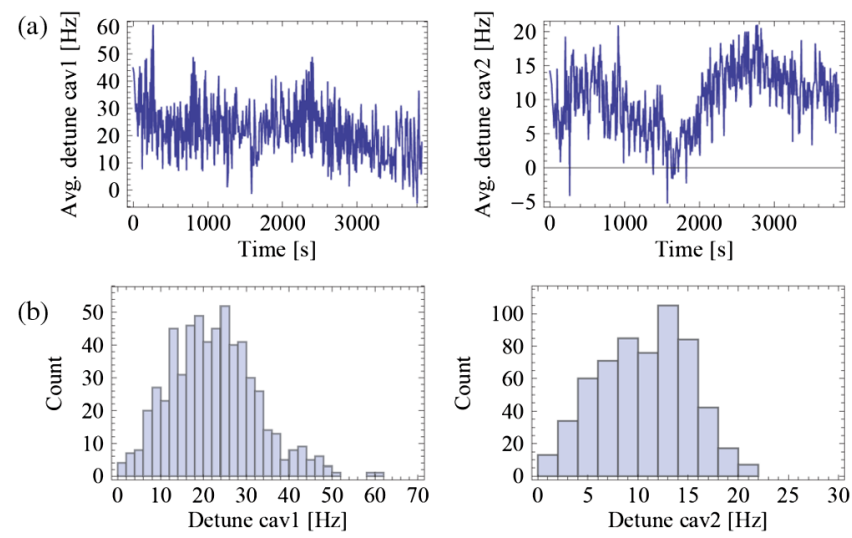

FIG. 9. (a) Detuning $[\mathrm{Hz}]$ of both cavities versus time [s] and (b) detuning histogram for both cavities during high $\mathrm{Q}_{\mathrm{L}}$ operation.

stability was sufficient so that the average detuning was smaller than the cavity bandwidths. With this a successful demonstration of very stable high $\mathrm{Q}_{\mathrm{L}}$ operation of both cavities was allowed, with stabilities comparable to nominal operation.

\section{AUTOMATED PKQL OPERATION}

Since the cavity gradient tilts and rf fluctuations induce transverse beam orbit changes [9], a stable beam acceleration requires flat cavity gradients during the beam transient. A further requirement of ILC is to operate all cavities (39 cavities per klystron) at 5\% below their respective quench limits over the whole flattop region of the pulse. This can only be achieved with matched conditions for all cavities. Since the beam loading is different for each cavity, the driving powers $\left(\mathrm{P}_{\mathrm{k}}\right)$ have to be adjusted individually. During the filling time of the pulse, no beam is present. In order to achieve a matched condition right after the filling of all cavities, also the loaded $\mathrm{Q}$ values $\left(\mathrm{Q}_{\mathrm{L}}\right)$ have to be adjusted individually. This operation mode is called $\mathrm{P}_{\mathrm{k}} \mathrm{Q}_{\mathrm{L}}$ operation. We have successfully demonstrated an automated procedure to engage in $\mathrm{P}_{\mathrm{k}} \mathrm{Q}_{\mathrm{L}}$ operation and maintaining a long-time $\mathrm{P}_{\mathrm{k}} \mathrm{Q}_{\mathrm{L}}$ operation [10,14]. The final operation parameters were $\mathrm{V}_{\text {Cav1 }}=16 \mathrm{MV} / \mathrm{m}, \mathrm{V}_{\mathrm{Cav} 2}=24 \mathrm{MV} / \mathrm{m}, \mathrm{Q}_{\mathrm{L}, \mathrm{Cav} 1}=9 \times 10^{6}$, $\mathrm{Q}_{\mathrm{L}, \text { Cav2 }}=3 \times 10^{6}$, a filling time of $410 \mu \mathrm{s}$, an average beam current of $6.4 \mathrm{~mA}$, and a beam pulse length of $615 \mu \mathrm{s}$. The virtual quench limits were defined to be 16.8 and $25.2 \mathrm{MV} / \mathrm{m}$, respectively, and were never exceeded.

To engage in automated setting procedure, the working points for the above-mentioned operation parameters first had to be determined in terms of $\mathrm{P}_{\mathrm{k}}$ and $\mathrm{Q}_{\mathrm{L}}$. Solving the cavity differential equation [15] yields the required power values during filling and flattop as shown in Eqs. (4) and (5):

$$
\begin{gathered}
P_{\text {fill }}=\frac{V_{\text {cav }}^{2}}{4 \frac{r}{Q} Q_{\mathrm{L}}\left[1+\exp \left(\frac{-\omega_{0} t_{\text {fill }}}{Q_{\mathrm{L}}}\right)-2 \exp \left(\frac{-\omega_{0} t_{\text {fill }}}{2 Q_{\mathrm{L}}}\right)\right]}, \\
P_{\text {flat }}=\frac{V_{\text {cav }}}{4 \frac{r}{Q} Q_{\mathrm{L}}}\left(1+\frac{\frac{r}{Q} Q_{\mathrm{L}} I_{\mathrm{b} 0}}{V_{\text {cav }}}\right)^{2},
\end{gathered}
$$

which hold for the constraints on flat flattops $\left(\frac{\mathrm{d} V_{\text {cav }}}{\mathrm{d} t}=0\right)$ and on resonance $(\Delta \omega=0)$. Since both cavities are driven by a single klystron, the power ratio of filling to flattop is the same. Thus, the $\mathrm{P}_{\mathrm{k}} \mathrm{Q}_{\mathrm{L}}$ working points for both cavities are determined as shown in Fig. $10\left(\mathrm{Q}_{\mathrm{L}, \mathrm{Cav} 1}=9.0 \times 10^{6}\right.$ and $\mathrm{Q}_{\mathrm{L}, \mathrm{Cav} 2}=3.0 \times 10^{6}$ ).

The procedure to engage in $\mathrm{P}_{\mathrm{k}} \mathrm{Q}_{\mathrm{L}}$ operation covers the following steps: (i) setting the $\mathrm{Q}_{\mathrm{L}}$ values using waveguide reflectors $\left(\mathrm{Q}_{\mathrm{L}}\right.$ control, automated), (ii) detuning compensation using piezotuners (automated), (iii) setting the cavity gradients by adjustment of the ratio of the variable hybrid and the feed-forward amplitude ( $\mathrm{P}_{\mathrm{k}}$ control, automated), (iv) phase compensation using waveguide phase shifters (automated), (v) turning on feedback (manually), (vi) turning on beam (manually), (vii) turning on beam compensation (manually), (viii) simultaneous extension of the beam pulse and cavity gradient flattop lengths (automated).

The manual steps were not automated because of machine protection concerns. A snapshot of the cavity 1 , cavity 2 , and vector-sum gradients during $\mathrm{P}_{\mathrm{k}} \mathrm{Q}_{\mathrm{L}}$ operation is shown in Fig. 11.

Figure 12 shows the gradient stabilities for cavities 1 and 2 versus time for a $800 \mathrm{~s}$ sample time span of the performed $1 \mathrm{~h}$ long-time operation with beam. The meanvector-sum stabilities were $\Delta A / \mathrm{A}_{\text {Cav1, mean }}=0.211 \%$ and $\Delta A / \mathrm{A}_{\mathrm{Cav} 2 \text {,mean }}=0.132 \%$ and the best $\Delta A / \mathrm{A}_{\mathrm{Cav} 1 \text {,best }}=$ $0.041 \%$ and $\Delta A / \mathrm{A}_{\text {Cav2,best }}=0.031 \%$.

Figure 13 shows the vector-sum gradient and phase stabilities during the beam transient versus time for the same $800 \mathrm{~s}$ sample time span. The vector-sum amplitude and phase stabilities are listed in Table III. These are comparable to the stabilities during nominal operation and hence allow a very stable actual $\mathrm{P}_{\mathrm{k}} \mathrm{Q}_{\mathrm{L}}$ operation, demonstrated for the first time here.
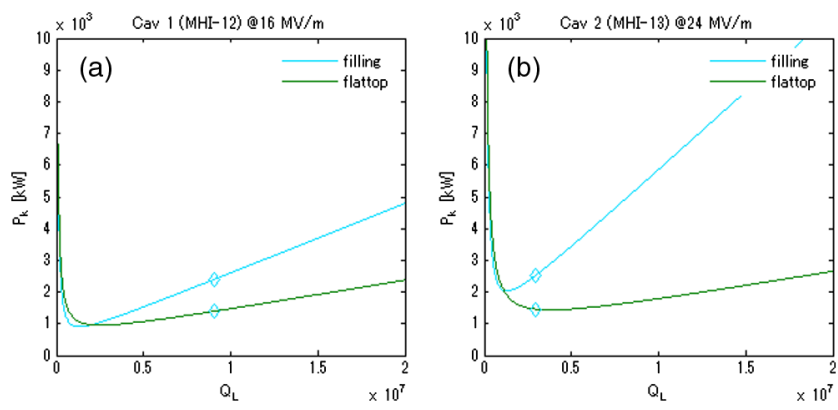

FIG. 10. Power (kW) versus $\mathrm{Q}_{\mathrm{L}}$ for (a) cavity 1 and (b) cavity 2 . The working points are marked by blue diamonds. 


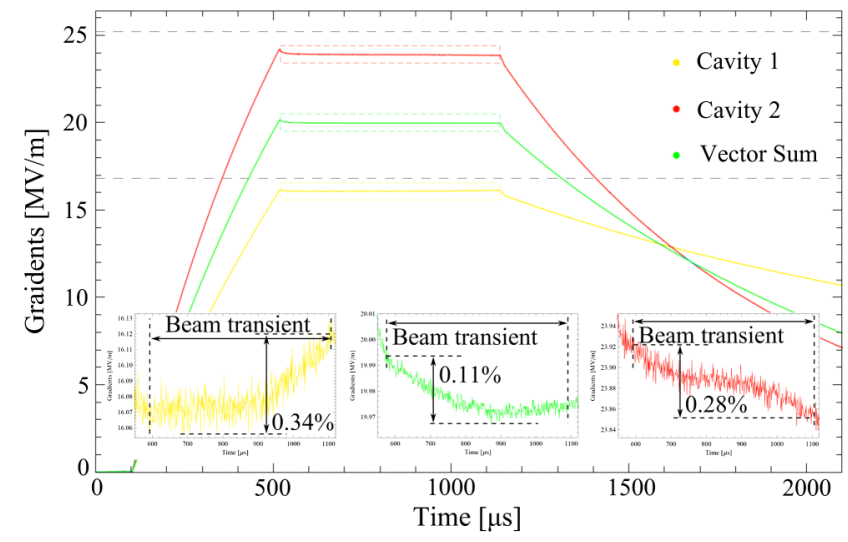

FIG. 11. Cavity 1 , cavity 2 , and the vector-sum gradients $(\mathrm{MV} / \mathrm{m})$ versus time $(\mathrm{s})$ during $\mathrm{P}_{\mathrm{k}} \mathrm{Q}_{\mathrm{L}}$ operation. The dashed lines indicate the virtual quench limits of 16.8 and $25.2 \mathrm{MV} / \mathrm{m}$, respectively.
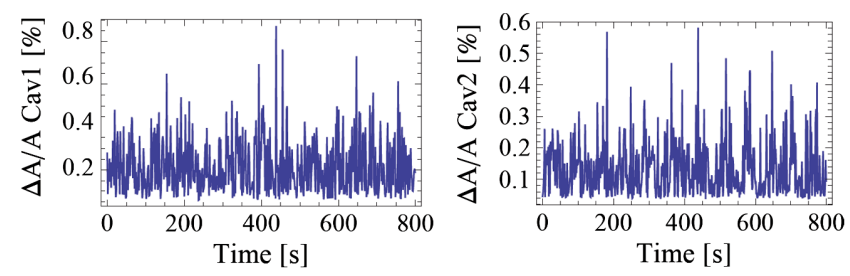

FIG. 12. Cavity 1 (left) and 2 (right) gradient stabilities during beam transient versus time $(\mathrm{s})$ in $\mathrm{P}_{\mathrm{k}} \mathrm{Q}_{\mathrm{L}}$ operation.
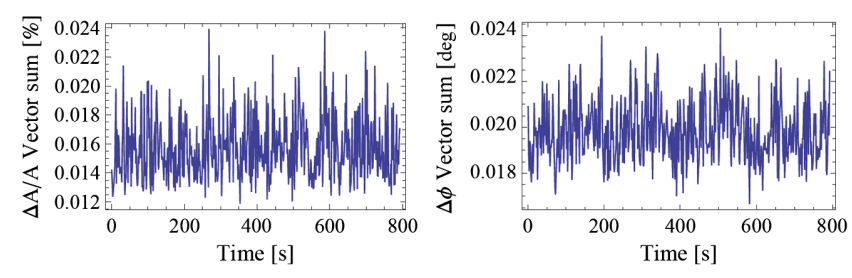

FIG. 13. Vector-sum gradient and phase stabilities during beam transient versus time (s).

The dominant source for the cavity as well as vector-sum stability fluctuations was a beam current fluctuation of about $20 \%$. Even under this condition, the average vectorsum stabilities, indicated as "mean" in Table III, were maintained. If the beam current could be controlled precisely and the matched condition maintained, the

TABLE III. Vector-sum amplitude and phase stabilities under $\mathrm{P}_{\mathrm{k}} \mathrm{Q}_{\mathrm{L}}$ operation (rms values).

\begin{tabular}{ll}
\hline \hline Beam & $6.1 \mathrm{~mA}$ \\
\hline Best vector sum $\Delta \mathrm{A} / \mathrm{A}$ & $0.009 \%$ \\
Mean vector sum $\Delta \mathrm{A} / \mathrm{A}$ & $0.016 \%$ \\
Best vector sum $\Delta \phi$ & $0.009^{\circ}$ \\
Mean vector sum $\Delta \phi$ & $0.019^{\circ}$ \\
\hline \hline
\end{tabular}

vector-sum stabilities would be as good as the ones indicated "best" in Table III for the whole time.

\section{SUMMARY AND OUTLOOK}

We have successfully demonstrated ILC-like nearquench-limit operations at KEK STF, using two superconducting nine-cell cavities driven by a single klystron. The rf phases of the cavities for on-crest beam acceleration were determined and set with a relative aberration of less than $3^{\circ}$. The cavity gradient measurement by the downconverted and digitized pickup signals was calibrated with an error of $3.8 \%$. An automated beam compensation was implemented, yielding vector-sum-gradient and phase stabilities for a long-time (in the scope of the QB project) nominal operation with beam of $\Delta \mathrm{A} / \mathrm{A}=0.009 \% \mathrm{rms}$ and $\Delta \phi=0.009^{\circ} \mathrm{rms}$. In a long-time high $\mathrm{Q}_{\mathrm{L}}$ operation $\left(\mathrm{Q}_{\mathrm{L}, \text { Cav1 }}=\mathrm{Q}_{\mathrm{L}, \text { Cav } 2}=2 \times 10^{7}, \mathrm{~V}_{\text {Cav1 }}=\mathrm{V}_{\text {Cav2 }}=20 \mathrm{MV} / \mathrm{m}\right)$ with beam the vector sum stabilities were $\Delta \mathrm{A} / \mathrm{A}=$ $0.011 \% \mathrm{rms}$ and $\Delta \phi=0.015^{\circ} \mathrm{rms}$. An automated $\mathrm{P}_{\mathrm{k}} \mathrm{Q}_{\mathrm{L}}$ setting procedure led to $\mathrm{V}_{\text {Cav1 }}=16 \mathrm{MV} / \mathrm{m}, \mathrm{V}_{\text {Cav2 }}=$ $24 \mathrm{MV} / \mathrm{m}$, both gradients $5 \%$ below their respective virtual quench limits, $\mathrm{Q}_{\mathrm{L}, \mathrm{Cav} 1}=9 \times 10^{6}, \mathrm{Q}_{\mathrm{L}, \mathrm{Cav} 2}=3 \times 10^{6}$. The vector-sum-gradient and phase stabilities for the long-time $\mathrm{P}_{\mathrm{k}} \mathrm{Q}_{\mathrm{L}}$ operation during the beam transient were $\Delta \mathrm{A} / \mathrm{A}=$ $0.009 \% \mathrm{rms}$ and $\Delta \phi=0.009^{\circ} \mathrm{rms}$, comparable to those under nominal operation.

Since $\mathrm{P}_{\mathrm{k}} \mathrm{Q}_{\mathrm{L}}$ operation demands the operation at very specific working points, it is sensitive to operation parameter deviations. Simulations of $\mathrm{P}_{\mathrm{k}} \mathrm{Q}_{\mathrm{L}}$ operation with ILC parameters showed that deviation limits were $+5.3 \%$ for the cavity driving power $\left(\mathrm{P}_{\mathrm{k}}\right),-11.6 \%$ for $\mathrm{Q}_{\mathrm{L}}$ values, $\pm 13.6^{\circ}$ for the cavity phase, and $-6.3 \%$ for the beam current. Exceeding those limits leads to cavity quenching. In the present study all LLRF parameter deviations were within these limits. Only the beam current fluctuation exceeded the limit. In the future an adaptive $\mathrm{P}_{\mathrm{k}} \mathrm{Q}_{\mathrm{L}}$ operation could be developed, which follows slow beam current changes or drifts. Since step motor positions of the waveguide reflectors cannot be adjusted in a pulse-to-pulse time span a sufficiently stable beam current is nonetheless essential.

[1] http://www.linearcollider.org.

[2] The International Linear Collider-Technical Design Report, CERN, FNAL, KEK, 2013.

[3] A. Kuramoto and H. Hayano, in Proceedings of the 4th International Particle Accelerator Conference, IPAC-2013, Shanghai, China, 2013 (JACoW, Shanghai, China, 2013), MOPME019, p. 509.

[4] S. Fukuda, in Proceedings of the 25th International Linear Accelerator Conference, LINAC-2010, Tsukuba, Japan (KEK, Tsukuba, Japan, 2010), MOP027, p. 112. 
[5] T. Miura et al., in Proceedings of the 2nd International Particle Accelerator Conference, San Sebastián, Spain (EPS-AG, Spain, 2011), MOPC155, p. 445.

[6] S. Michizono et al., in Proceedings of the 2nd International Particle Accelerator Conference, San Sebastián, Spain (Ref. [5]), MOPC157, p. 451.

[7] E. Vogel, Phys. Rev. ST Accel. Beams 10, 052001 (2007).

[8] C. Pagani et al., in Proceedings of SRF2011, Chicago (JACoW, Geneva, 2011), THIOA01, p. 615.

[9] K. Kubo, Effect of Cavity Tilt and RF Fluctuation to Transverse Beam Orbit Change in ILC Main Linac (KEK, Tuskuba, 2010).

[10] M. Omet et al., in Proceedings of the 4th International Particle Accelerator Conference, IPAC-2013, Shanghai, China, 2013 (Ref. [3]), WEPME013, p. 2950.

[11] K. L. F. Bane, C. Adolphsen, and C. Nantista, in Proceedings of the 16th IEEE International Pulsed Power
Conference, Albuquerque, NM (IEEE, Piscataway, NJ, 2007), WEPMS037, p. 2424.

[12] J. Branlard, in Proceedings of LINAC2012, Tel-Aviv (JACoW, Geneva, 2012), THPB085, p. 1023.

[13] M. Omet et al., Development and Application of a Frequency Scan-based and a Beam-based Calibration Method for the LLRF Systems at KEK STF, in Proceedings of the 9th Annual Meeting Particle Accelerator Society Japan, Osaka, 2012 (Particle Accelerator Society of Japan, Tokyo, 2012), PASJ2012, p. 265.

[14] M. Omet, T. Matsumoto, S. Michizono, and T. Miura, Demonstration of RF Stabilities in STF 9-cell Cavities Aiming for the Near Quench Limit Operation, in Proceedings of SRF13, Paris (JACoW, Geneva, 2013), THIOC04, p. 865.

[15] T. Schilcher, Ph.D. thesis, Universität Hamburg, 1998. 